\title{
Recherches sociographiques
}

\section{Madeleine FERRON et Robert CLICHE, Quand le peuple fait la} loi

\section{Vincent Lemieux}

Volume 13, numéro 3, 1972

Idéologies et politiques étudiantes

URI : https://id.erudit.org/iderudit/055595ar

DOI : https://doi.org/10.7202/055595ar

Aller au sommaire du numéro

Éditeur(s)

Département de sociologie, Faculté des sciences sociales, Université Laval

ISSN

0034-1282 (imprimé)

1705-6225 (numérique)

Découvrir la revue

Citer ce compte rendu

Lemieux, V. (1972). Compte rendu de [Madeleine FERRON et Robert CLICHE, Quand le peuple fait la loi]. Recherches sociographiques, 13(3), 413-414.

https://doi.org/10.7202/055595ar d'utilisation que vous pouvez consulter en ligne.

https://apropos.erudit.org/fr/usagers/politique-dutilisation/ 
Il n'en subsiste pas moins, par delà des faiblesses qu'explique fort bien au demeurant la jeunesse des sciences politique et administrative comme disciplines et le petit nombre au Québec d'études antérieures dans ce secteur des sciences sociales, que l'ouvrage d'André Gélinas vient incontestablement apporter une pierre, une des toutes premières, à l'édification d'une meilleure connaissance des rapports administrationenvironnement, rapports dont le parlement est évidemment un des interlocuteurs privilégiés.

\section{Alain Baccigalupo}

Département de science politique, Université Laval.

Madeleine FERRon et Robert ClICHE, Quand le peuple fait la loi. La loi populaire à Saint-Joseph de Beauce, Montréal, Hurtubise, 1972, 157 p.

Ce livre nous fait connaître Robert Cliche, mieux que ses gestes d'homme politique ou d'homme de loi. Il nous fait aussi mieux connaître la Beauce. La présence discrète de Madeleine Ferron, dont on présume qu'elle a dû arrondir une phrase, suggérer une transition, ajoute au charme de l'ouvrage. Il faut espérer, comme on nous l'annonce, que cette collaboration se poursuivra. Nous pourrons arriver ainsi à nous mieux comprendre, loin de la clameur des colloques, des discussions à la TV, ou des campagnes électorales.

« La loi * que fait le peuple, il faut l'entendre dans un sens large. C'est, nous dit-on, " l'ensemble des conventions qu'établissent, entre eux, les membres d'un groupe déterminé, dans un endroit déterminé, pour régir leurs relations * (pp. 15-16). L'individu ne la comprend pas, il y est " compris". En se fondant sur la tradition orale les deux auteurs nous présentent successivement la loi populaire qui régit la famille paternelle, qui s'observe dans les funérailles et à propos des trépassés, qui règle le ménage conjugual et l'adoption, qu'on trouve dans le droit contractuel et dans le droit punitif, qui s'applique aux querelles et aux batteries, qui se retrouve aussi à propos des servitudes (cours d'eau), de la chasse et de la pêche, dans les brocards populaires, à l'occasion de l'élection des maires et des marguilliers, et enfin dans les relations avec les quêteux et les nomades ainsi que dans les fonctions des jurés.

Les folkloristes, plus que les sociologues ou les ethnologues, semblent avoir guidé Madeleine Ferron et Robert Cliche. André Varagnac, dans sa Civilisation traditionnelle et genres de vie, apparaît comme leur auteur préféré. Ils se fondent sur son autorité pour ne pas s'employer à délimiter trop strictement dans le temps et dans l'espace l'observance des traditions populaires - ce qui leur a été reproché. Mais le reproche porte à faux, étant donné ce qu'ont voulu faire les deux auteurs: des tableaux plus littéraires que scientifiques, mais non moins fidèles, qui nous éveillent à la bonne humeur mais aussi à la sagesse des gens de la Beauce.

De même qu'ils n'ont pu résister à l'inclure, je ne puis résister à citer le passage suivant des deux auteurs, qui me semble illustrer parfaitement leur authenticité, leur style, les tentations auxquelles ils succombent fort heureusement :

"C'est la première fois, à notre connaissance, que la Vierge se soit permis de jurer. Notre informateur, répondant au surnom de "Ti-Petit ", nous raconte 
qu'un jour, alors qu'il bûchait dans sa sucrerie, il vit apparaître la Vierge Marie qui s'avança "toute dorée de lumière " revêtue de sa longue tunique * couleur de firmament ». Il trimait depuis le matin, * abattait l'eau > de fatigue. S'essuyant les yeux pour mieux y voir, prêt à défaillir de surprise et d'épuisement, il la vit se pencher vers lui et lui dire avec douceur : " slack, Ti-Petit, slack, Calvaire !* (p. 48)

On voit rire Robert Cliche, de tous les traits de son visage. Mais le conseil de la Vierge n'est pas seulement cocasse, il est fort significatif de ce que certains appellent nos contradictions, d'autres notre culture. Une Vierge qui parle « joual» et qui "sacre » pour mieux se faire entendre, n'est-ce pas un merveilleux révélateur? Une analyse seconde des données que nous présentent Madeleine Ferron et Robert Cliche en décelerait bien d'autres. Elle pourrait montrer comment les pratiques sociales de la Beauce étaient interprétées par les représentations religieuses, et celles-ci par celles-là, en métaphore les unes des autres : l'avocat qui plaidait devant les jurés exigeait des honoraires plus élevés parce "qu'une grand-messe coûte plus cher qu'une basse messe * (p. 151) ; mais quand, devant le corps d'un défunt, on fait chevaucher avec aisance les deux versets de l'Ave Maria, on dit * un chapelet en bardeaux * (p. 45).

On regrette un peu que la politique tienne peu de place dans ce petit livre, et surtout qu'elle soit limitée aux élections municipales et aux élections de marguilliers. Pourtant Robert Cliche a certainement beaucoup à dire sur cette activité pour laquelle la Beauce est justement réputée, et à laquelle il a consacré une bonne partie de sa vie. Notons ce jugement qui est porté sur les créditistes, et qui vaut bien des analyses sociologiques :

* Alors qu'au début, les créditistes luttaient contre l'oligarchie existante, maintenant ils contestent cette société nouvelle qui a chambardé les traditions, sans apporter, en compensation, l'équilibre promis. Le partage du revenu national est le rêve du retour à la vie communautaire et égalitaire qui était la leur, dont ils contrôlaient les rouages. Ils opposent le * bon sens » (ou reflexion personnelle) au nivellement des esprits par l'instruction. Qu'on soigne la communauté entière, mais que ce soit l'individu qui paie avec l'argent de l'État. Le Crédit Social mène une lutte obscure et maladroite contre la dépersonnalisation. » (p. 23)

On attend donc la suite, en songeant qu'il faudrait nous dépêtrer un peu plus de nos fonctions, de nos manuels et de nos techniques respectables, pour aller retrouver simplement les gens, sur leur terrain.

Département de science politique,

Vincent LEMIEUX

Université Laval.

Christian Morissonneau, La Société de Géographie de Québec. 1877-1970, Québec, Presses de l'Université Laval, 1971.

Les personnages scientifiques importants des dix-huitième et dix-neuvième siècles, l'activité et le rayonnement des sociétés dites savantes figurent parmi les grands a laissés pour compte » de notre historiographie québécoise. N'est-ce pas là un refrain connu ? 